\title{
Entomological Surveillance Associated with Human Zika Cases in Miri Sarawak, Malaysia
}

\author{
Roziah Ali, ${ }^{1,2 *}$ Ruziyatul Aznieda Azmi, ${ }^{1}$ Nazni Wasi Ahmad, ${ }^{1}$ Azahari Abd Hadi, ${ }^{1}$ Khairul Asuad Muhamed, ${ }^{1}$ Rosilawati Rasli, ${ }^{1}$ \\ Cheong Yoon Ling, ${ }^{1}$ Henry Anak Chua, ${ }^{3}$ Kiew Lian Wan, ${ }^{2}$ and Han Lim Lee ${ }^{1}$ \\ ${ }^{1}$ Medical Entomology Unit, Institute for Medical Research, WHO Collaborating Centre for Vectors, Kuala Lumpur, Malaysia; ${ }^{2}$ School of Biosciences \\ and Biotechnology, Faculty of Science and Technology, Universiti Kebangsaan Malaysia, Selangor, Malaysia; ${ }^{3}$ Miri District Health Office, Jalan \\ Temenggong Oyong Lawai Jau, Miri, Malaysia
}

\begin{abstract}
Two confirmed human cases of Zika virus (ZIKV) were reported in the district of Miri, Sarawak, in 2016. Following that, a mosquito-based ZIKV surveillance study was conducted within 200-m radius from the case houses. Mosquito surveillance was conducted using five different methods, that is, biogents sentinel mosquito (BG) sentinel trap, modified sticky ovitrap, resting catch, larval surveillance, and conventional ovitrap. A total of 527 and 390 mosquito samples were obtained from the case houses in two localities, namely, Kampung Lopeng and Taman Shang Ri La, Miri, Sarawak, respectively. All mosquitoes collected were identified, which consisted of 11 species. Aedes albopictus, both the adult and larval stages, was the dominant species. Resting catch method obtained the highest number of adult mosquitoes (67\%), whereas ovitrap showed the highest catch for larval mosquitoes (84\%). Zika virus was detected in both adults and larvae of Ae. albopictus together with adults of Culex gelidus, and Culex quinquefasciatus using the real-time reverse transcriptase polymerase chain reaction (PCR) technique. It was noteworthy that Ae. albopictus positive with ZIKV were caught and obtained from four types of collection method. By contrast, Cx. gelidus and Culex quinquefasciatus adults collected from sticky ovitraps were also found positive with ZIKV. This study reveals vital information regarding the potential vectors of ZIKV and the possibility of transovarian transmission of the virus in Malaysia. These findings will be essentials for vector control program managers to devise preparedness and contingency plans of prevention and control of the arboviral disease.
\end{abstract}

\section{INTRODUCTION}

Zika virus (ZIKV) is a mosquito-borne pathogen that belongs to the genus of Flavivirus of the family Flaviviridae. It was isolated from Aedes africanus mosquito that were collected from the same locality of the first isolation from monkey in 1947 in Uganda. ${ }^{1}$ Individuals with mild infection of ZIKV commonly show symptoms of fever, rash, join pain, and conjunctivitis (red eyes), which are similar to characteristics and symptoms of dengue infections. ${ }^{2}$ However, during Zika outbreak in Brazil, studies revealed that ZIKV infections have been associated with microcephaly and neurological disorder cases among newborns. ${ }^{3}$

In 1969, Marchette et al. ${ }^{4}$ reported the first isolation of ZIKV from Malaysian Aedes aegypti. They collected mosquitoes in a wide variety of habitats that included rural, urban areas and different forest types. In addition, there were several cases of serological evidence of ZIKV collected from the aborigine population during the $1960 \mathrm{~s}^{5}$ There was one confirmed case with clinical evidence of Zika fever reportedly found in a German after visiting Sabah, Malaysia, in $2014 .{ }^{6}$ Following that, a massive outbreak of ZIKV infection was reported in Singapore in 2016. Subsequently, ZIKV infection of individuals with a travel history to Singapore was reported in Malaysia, including two cases in Miri, Sarawak.

Because ZIKV has been isolated from Malaysian Ae. aegypti in $1969,{ }^{4}$ there is a high possibility of the circulation of this virus in the environment. There is also a higher chance of ZIKV to be isolated from Aedes mosquitoes in Malaysia. However, it is also important to screen the virus in other vector mosquitoes. A study by Brazilian researchers suggested that the common house mosquito $C x$. quinquefactiatus could be involved in the

\footnotetext{
* Address correspondence to Roziah Ali, Medical Entomology Unit, Institute for Medical Research, WHO Collaborating Centre for Vectors, Jalan Pahang, Kuala Lumpur 50588, Malaysia. E-mail: roziah@ imr.gov.my
}

transmission of the virus as it was found infected with ZIKV genome fragments. ${ }^{7}$ Furthermore, a laboratory study in China demonstrated that Culex quinquefasciatus has a potential role as a vector in transmitting $\mathrm{ZIKV}^{8}$ Nevertheless, there were laboratory studies that also demonstrated the Culex are not competent vectors for ZIKV transmission. ${ }^{9-11}$

Thus, this study was conducted to sample mosquito adults and larvae from the Miri district in Sarawak, Malaysia, using different types of traps and techniques. The traps were placed at the surrounding area of the patient houses prior within a 200- to $300-\mathrm{m}$ radius before mosquito collection. The real-time reverse transcriptase PCR technique was then conducted to screen ZIKV from these field-caught mosquitoes to determine their potential as vector for ZIKV. Data acquired will be essential for early preventive and control measures for the outbreak of Zika.

\section{MATERIALS AND METHODS}

Site selection and mosquito collection. The study was conducted in the Miri district of Sarawak, Malaysia, which has an estimated population of 67,000 households (Department of Statistics Malaysia, 2010). Based on the reported confirmed case of Zika infection, two sampling sites, Kg. Lopeng (N 4 ${ }^{\circ} 22^{\prime}$ 41.455"E 113 $\left.59^{\prime} 23.593^{\prime \prime}\right)$ and Shang Ri La (N 4¹9'47.622"E $\left.113^{\circ} 58^{\prime} 40.602^{\prime \prime}\right)$, were selected (Figure 1).

Five mosquito collection methods were used during the study, namely, BG sentinel trap, modified sticky ovitrap, resting catch, larval surveillance, and conventional ovitrap. A total of 100 modified sticky ovitraps and 30 conventional ovitraps were placed in 50 households indoor and outdoor within a $200-$ to $300-\mathrm{m}$ radius from each case house, respectively. For modified sticky ovitrap, the standard ovitrap container was improvised before catching the adult mosquitoes. A small pit was made at the side of the container to prevent overflowed water from flushing away the specimens, especially during heavy rains. A sticky sheet $(21 \mathrm{~cm} \times 6 \mathrm{~cm})$ was placed at the inner wall of the container beforehand and 


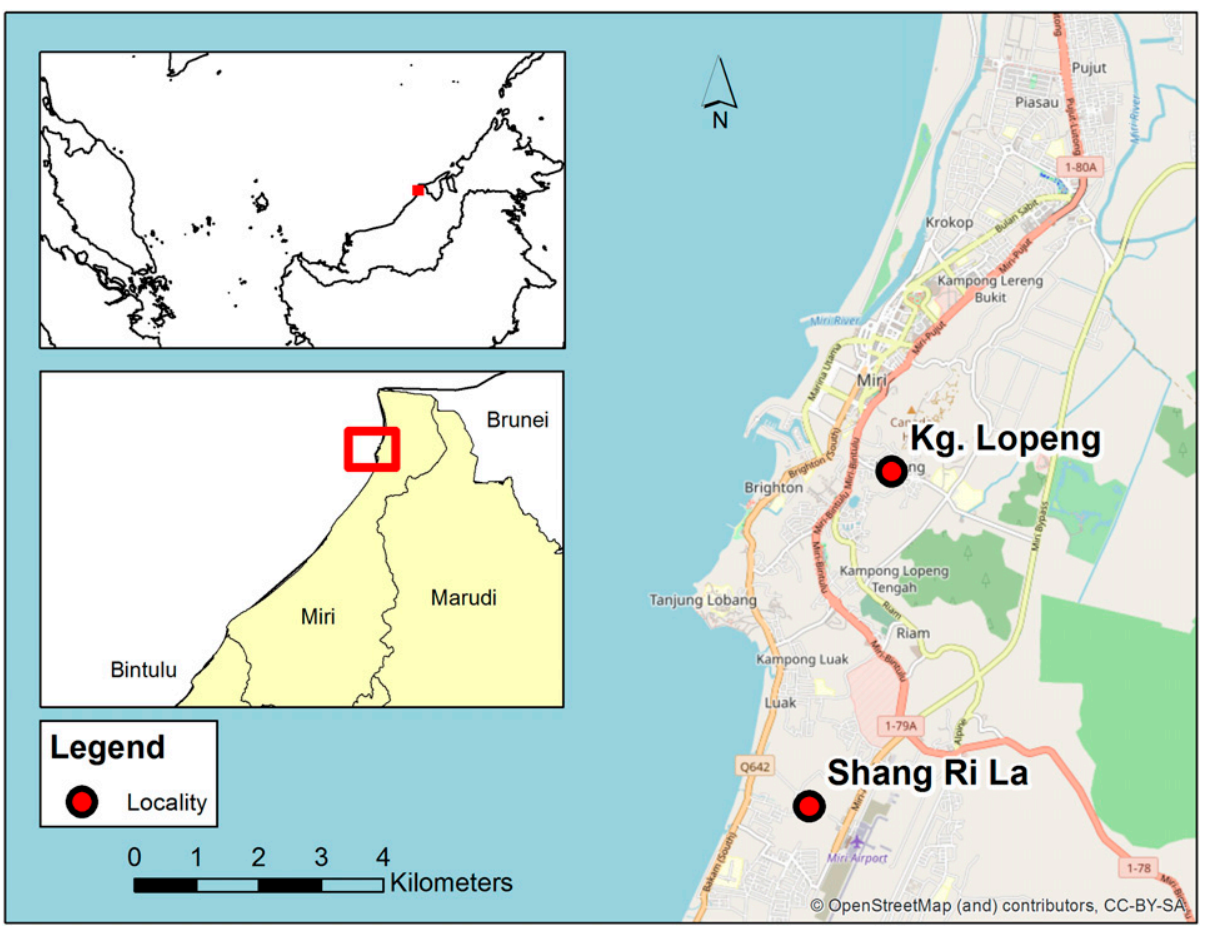

FIGURE 1. Study site locations: Kg. Lopeng and Shang Ri La, Miri, Sarawak. (Source of image: OpenStreetMap).

filled up with water. In this study, "indoor" refers to any parts of the premise that are directly under the roof, whereas "outdoor" refers to the areas outside the roof but within the immediate vicinity of the premise.

BG sentinel traps were placed near to the infected case house for 14 hours, that is, 5:00 AM to 7:00 PM Adult mosquito collection was also conducted by the resting catch method using aspirators at mosquito resting sites such as bushes, trees, potted plants, and wall. Resting mosquitoes were collected using electrically operated aspirator into clean paper cups, before the samples were transported back to the laboratory for processing. Blood-fed mosquitoes were allowed to digest the blood for 2-3 days before being processed.

Larval sample collection was carried out within a 200-m radius from the case-infected area. All potential indoor and outdoor mosquito-breeding sites were inspected. Larvae and pupae were collected using pipettes and placed in separate labeled containers. Important information such as the type of habitat (natural or artificial), number of samples, and stages of life cycle were recorded on prescribed forms.

Specimen identification and transportation. Adult mosquitoes collected were identified morphologically using standard taxonomy keys. Later, they were sorted out and pooled into groups according to the species, sex, locations, and type of collection method. However, mosquito specimens obtained from modified sticky ovitrap were processed individually. All specimens were kept in a cold box at sites and during transportation before virus detection process. For conventional ovitrap and larval surveillance specimens, all immature stages were brought back to the laboratory and bred to adulthood before species identification.

Virus detection in mosquito pool. Pooled mosquitoes (maximum of 25 per pool) were homogenized with $300 \mu \mathrm{L}$ of Minimum Essential Media Eagle solution using sterile pestle on ice. In total, $200 \mu \mathrm{L}$ of homogenate was used for the RNA extraction step using the QIAamp RNA Viral Kit (Qiagen, Heiden, Germany) following the manufacturer's protocol. The final RNA pellet was dissolved in $60 \mu \mathrm{L}$ of the provided buffer AVE and stored at $-80^{\circ}$ before virus screening. ZIKV detection was performed using real-time reverse transcription-PCR (RT-PCR) assay. RNA was amplified using the iTaq ${ }^{\mathrm{TM}}$ Universal Probes One-Step Kit in the BioRad CFX96 Touch RealTime PCR (Bio-rad Laboratories, Hercules, CA) machine in a $20-\mu \mathrm{L}$ reaction volume containing $5 \mu \mathrm{L}$ of extracted RNA, $0.5 \mu \mathrm{L}$ of iScript advanced reverse transcriptase, $10 \mu \mathrm{M}$ of each primers, and $25 \mu \mathrm{M}$ of fluorogenic probe, and addition of

TABLE 1

Collection of mosquitoes in $\mathrm{Kg}$

\begin{tabular}{|c|c|c|c|c|}
\hline \multirow[b]{3}{*}{ Species } & \multicolumn{4}{|c|}{ Collection site } \\
\hline & \multicolumn{2}{|c|}{ Kg Lopeng } & \multicolumn{2}{|c|}{ Shang Ri La } \\
\hline & $\begin{array}{c}\text { No. } \\
\text { collected }\end{array}$ & $\%$ & $\begin{array}{l}\text { No. } \\
\text { collected }\end{array}$ & $\%$ \\
\hline Aedes aegypti & 10 & 1.9 & 13 & 3.3 \\
\hline Aedes albopictus & 423 & 80.3 & 344 & 88.2 \\
\hline Aedes butleri & 3 & 0.6 & 2 & 0.5 \\
\hline Aedes niveus & 3 & 0.6 & 0 & 0.0 \\
\hline Aedes sp. & 1 & 0.2 & 5 & 1.3 \\
\hline Anopheles sundaicus & 1 & 0.2 & 0 & 0.0 \\
\hline Culex gelidus & 19 & 3.6 & 0 & 0.0 \\
\hline Culex pseudovishnui & 13 & 2.5 & 0 & 0.0 \\
\hline Culex quinquefasciatus & 42 & 8.0 & 25 & 6.4 \\
\hline Culex sitiens & 5 & 0.9 & 0 & 0.0 \\
\hline Culex sp. & 1 & 0.2 & 0 & 0.0 \\
\hline Culex tritaeniorhynchus & 2 & 0.4 & 0 & 0.0 \\
\hline Culex whitmorei & 2 & 0.4 & 0 & 0.0 \\
\hline Unknown (damaged specimens) & 2 & 0.4 & 1 & 0.3 \\
\hline Total & 527 & & 390 & \\
\hline
\end{tabular}

Lopeng and Shang Ri La, Miri, Sarawak. 


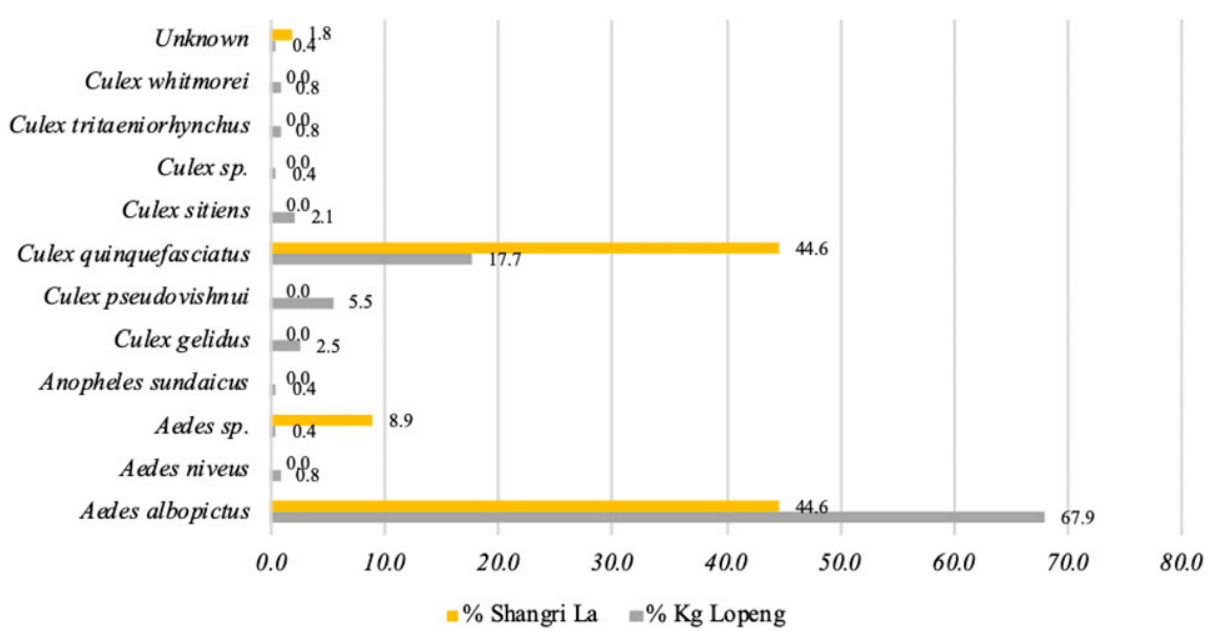

FIGURE 2. Percentage of adult mosquitoes captured in Kg. Lopeng and Shang Ri La, Miri, Sarawak. This figure appears in color at www.ajtmh.org.

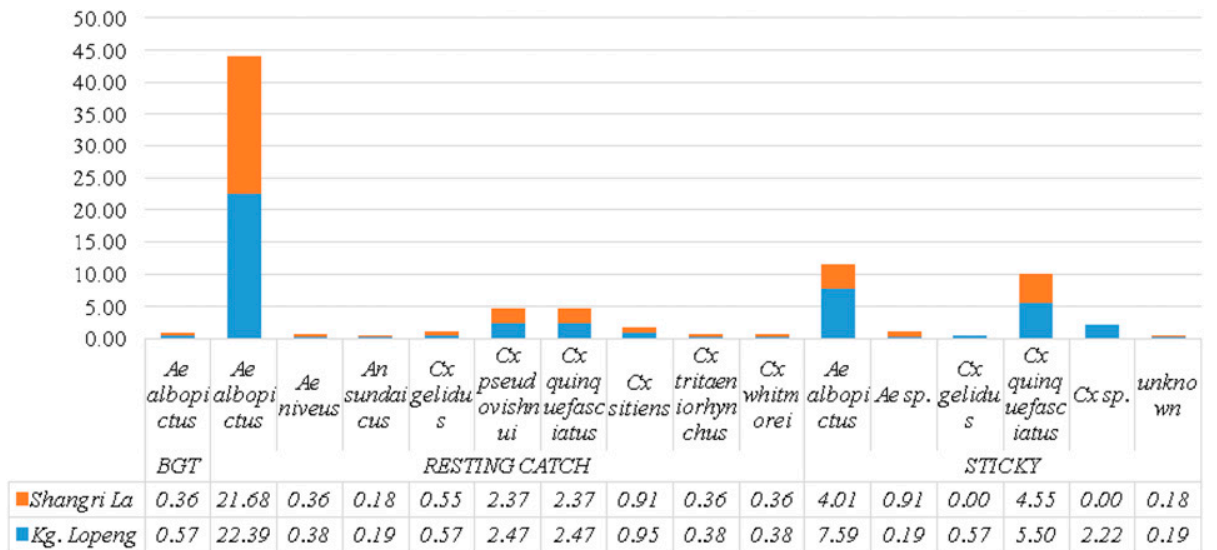

FIGURE 3. Percentage of adult mosquitoes captured based on methods. This figure appears in color at www.ajtmh.org.

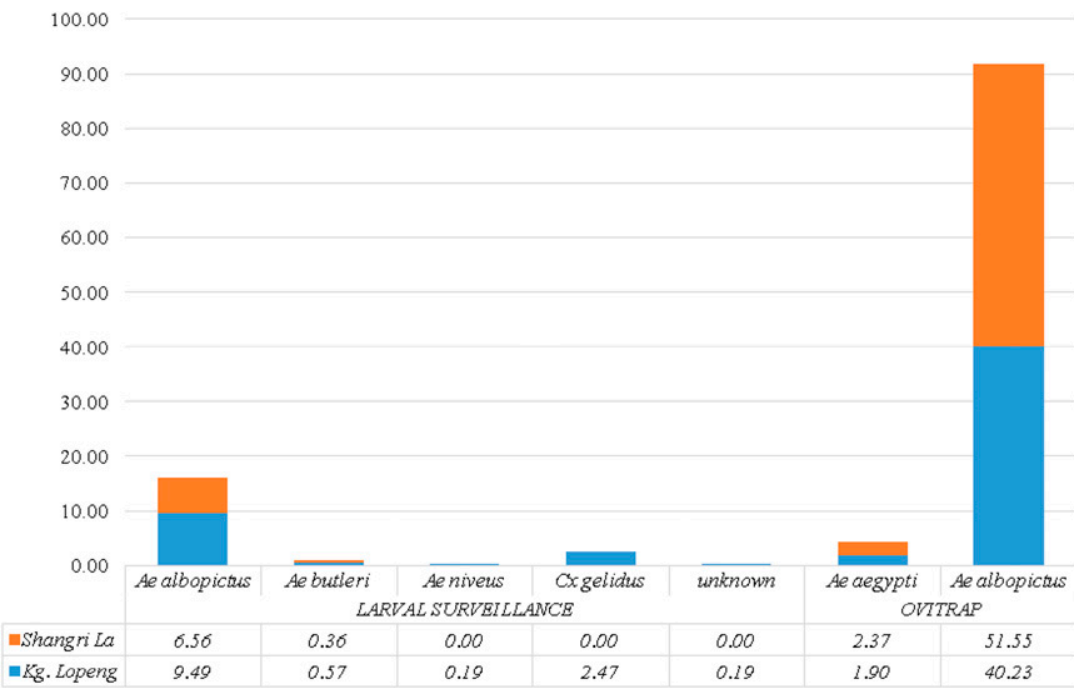

FIGURE 4. Total percentage of larvae collected in Kg. Lopeng and Shang Ri La, Miri, Sarawak, by larval surveillance and ovitrapping. This figure appears in color at www.ajtmh.org. 
nuclease-free water. The primers, probe, and PCR cycles were used as described by Lanciotti et al. ${ }^{2}$

Data analysis. The data were analyzed using descriptive statistics for comparison between numbers of mosquitoes based on collection methods and locations. Poisson regression analysis was used to calculate the significance of sampling methods from both locations. The virus infection rate was calculated with PooledlnfRate, version 4.0 by Biggerstaff. The add-in application in Microsoft Excel (Microsoft Excel for Windows, Redmond, WA) computes the IR using data from pooled samples even though the pool sizes were different from each other. The IR values were calculated by both minimum infection rates (MIRs) and maximum likelihood estimation. Locations of the mosquito samples with ZIKV detection were mapped using the QGIS: QGIS Geographical Information System (2019).

\section{RESULTS}

Mosquito species composition. A total of 527 and 390 mosquitoes were collected from $\mathrm{Kg}$. Lopeng and Shang Ri La, respectively, with the total captured comprising 11 species, that is, Aedes aegypti, Aedes albopictus, Aedes butleri, Aedes niveus, Anopheles sundaicus, Culex gelidus, Culex pseudovishnui, $\mathrm{Cu}$ lex quinquefasciatus, Culex sitiens, Culex tritaeniorhynchus, and Culex whitmorei (Table 1). Our results showed a high number of adult $\mathrm{Ae}$. albopictus captured in Kg. Lopeng (161, 67.9\%), whereas $A e$. albopictus and $C x$. cuinquefasciatus shared the abundance $(25,44.6 \%)$ from Shang Ri La. Furthermore, Cx. quinquefasciatus was recorded as the second highest number of predominant mosquito collected from the sites (Figure 2). This study also demonstrated that the resting catch method captured the most numbers of adult mosquito species compared with the modified sticky ovitrap and BG Sentinel trap, with nine species, five species, and one species, respectively. In addition, the resting catch method recorded the highest number of Ae. albopictus adult in both locations (Figure 3).

For larval collection, a total of 624 mosquito larvae comprising six species from two genera were obtained from the study sites. Ae. albopictus represented the highest number of collected larvae from both $\mathrm{Kg}$. Lopeng and Shang Ri La with 262 (90.34\%) and $317(95.5 \%)$, respectively. This study also reveals that ovitrapping method is more effective than the larval surveillance method. Moreover, this study also revealed that $A$ e. albopictus larvae were the predominant species collected through larval surveillance and conventional ovitrap (Figure 4).

Statistical analysis using Poisson regression showed that there is a significant difference between sampling methods used in this study, with a $P$-value $<0.001$, whereas the differences between locations were not significant, with a $P$-value $=0.914$.
Zika virus detection. Zika virus was detected from mosquitoes caught in both study locations. The MIRs of ZIKV were calculated based on mosquito species and locations (Table 2). It was noteworthy that mosquitoes positive with ZIKV were obtained from four types of collection method, which include adult and larvae samples. Remarkably, only Ae. albopictus species was positively screened with ZIKV from the most sampling methods ie; resting catch, larval surveillance, and ovitrap methods. Distribution of the positive mosquitoes in $\mathrm{Kg}$. Lopeng and Shang Ri La was mapped as in Figures 5 and 6, respectively.

\section{DISCUSSION}

After two confirmed human-infected cases of Zika were reported, mosquito composition in both localities was determined through the use of various collection techniques. In this study, Ae. albopictus was found to constitute the highest number of caught mosquito species for both adult and larvae stages. The abundance of Ae. albopictus indicates that the species is widespread and commonly found in urban and suburban areas of the sampling locations. Kg. Lopeng is categorized as a residential area located at the suburbs of the city and consists of traditional and terrace houses. This area includes workers' quarters that were built from wooden and cement walls with a poor drainage system. This sampling area is covered with natural vegetations and secondary forest. By contrast, Shang Ri La is considered as a new planned housing area comprising terrace and semidetached houses. Good drainage system at the area was observed during the visit. Similar findings were reported by another study ${ }^{12}$ where the authors found high density of Ae. albopictus in urban areas in Sarawak using ovitrap surveillance. Furthermore, Ae. albopictus is well known as a semidomestic breeder that can be found in artificial and natural containers. ${ }^{13,14}$ Domestic animals were also observed at the surrounding area of both locations in Kg. Lopeng and Shang Ri La. Moreover, domestic animals such as cats, dogs, and chickens can be found at both locations, which could contribute as a potential optimal breeding habitat for Ae. albopictus as it is both zoophilic and anthropophilic.

Ae. aegypti and $A$ e. albopictus have been long incriminated as ZIKV vectors worldwide. ${ }^{15-17}$ A study by Marchette et al. ${ }^{4}$ has reported that ZIKV was successfully isolated from $A e$. aegypti collected in Peninsular Malaysia resulting in the species being declared as a primary vector for ZIKV in Malaysia. However, in this study, we found that ZIKV was detected only in $A e$. albopictus mosquitoes. Even though ZIKV transmission through Ae. albopictus is unknown in Malaysia, several studies $^{18,19}$ have suggested that $A e$. albopictus is a vector as

TABLE 2

Result of Zika virus by locality and mosquito species showing the maximum likelihood estimation (MLE) and minimum infection rate (MIR) based on the total number of pools and individuals tested

\begin{tabular}{|c|c|c|c|c|c|c|c|}
\hline Stage & Collection sites & Mosquito species & No. of individuals & No. of positive pools & MLE & MIR & Type of methods \\
\hline \multirow[t]{5}{*}{ Adult } & \multirow[t]{3}{*}{ Kg. Lopeng } & Aedes albopictus & 161 & 5 & 33.22 & 31.06 & Sticky trap and resting catch \\
\hline & & Culex quinquefasciatus & 42 & 1 & 23.81 & 23.81 & Sticky trap \\
\hline & & Culex gelidus & 6 & 1 & 166.67 & 166.67 & Sticky trap \\
\hline & \multirow{2}{*}{ Shang Ri La } & Aedes albopictus & 25 & 1 & 40.83 & 40 & Sticky trap \\
\hline & & Culex quinquefasciatus & 25 & 2 & 153.85 & 153.85 & Sticky trap \\
\hline \multirow[t]{2}{*}{ Larvae } & Kg. Lopeng & Aedes albopictus & 262 & 3 & 7.65 & 7.63 & Larval surveillance \\
\hline & Shang Ri La & Aedes albopictus & 319 & 3 & 9.72 & 9.4 & Ovitrap and larval surveillance \\
\hline
\end{tabular}




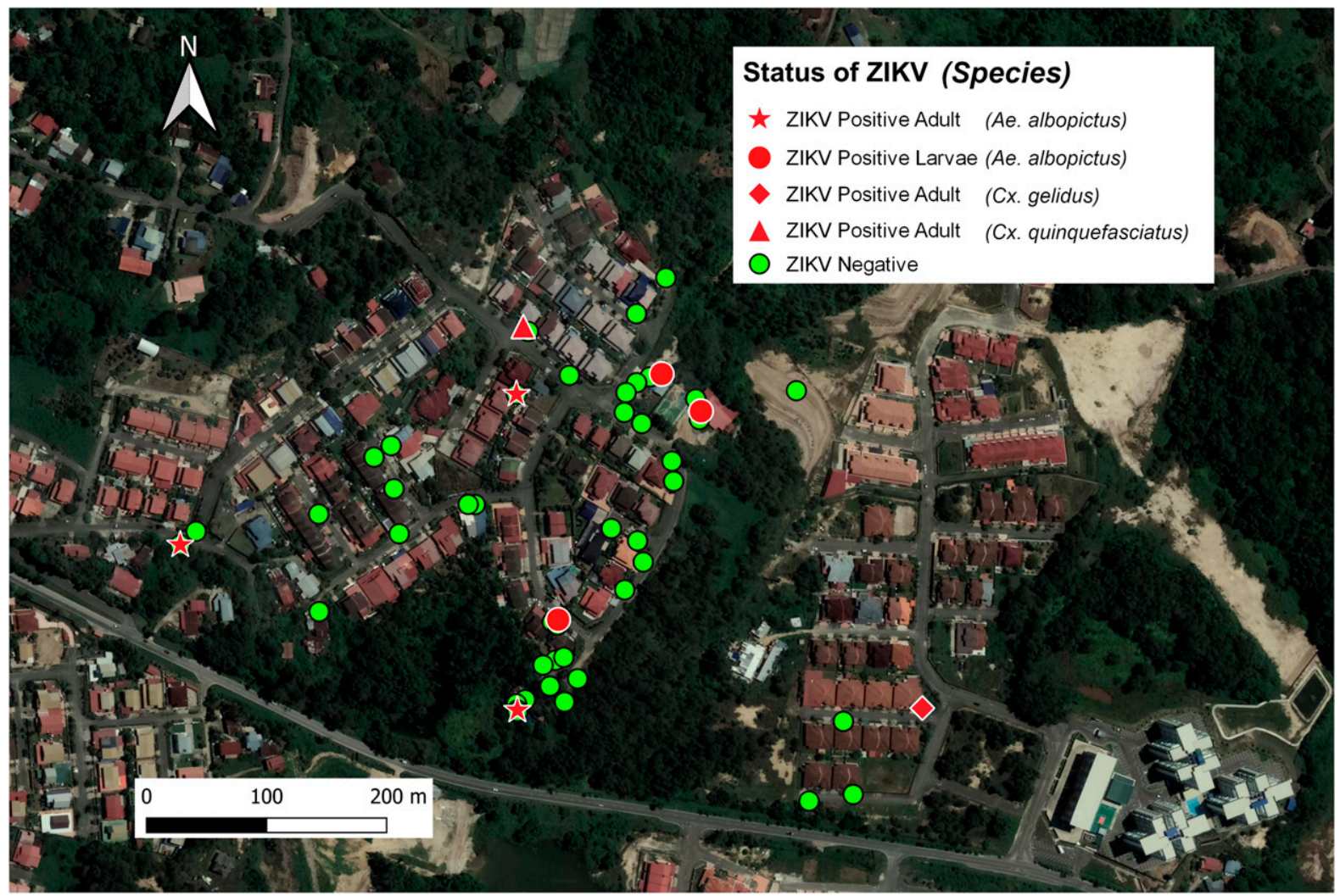

FIGURE 5. Geographical distribution of mosquito species with regard to Zika virus detection in Kg. Lopeng.

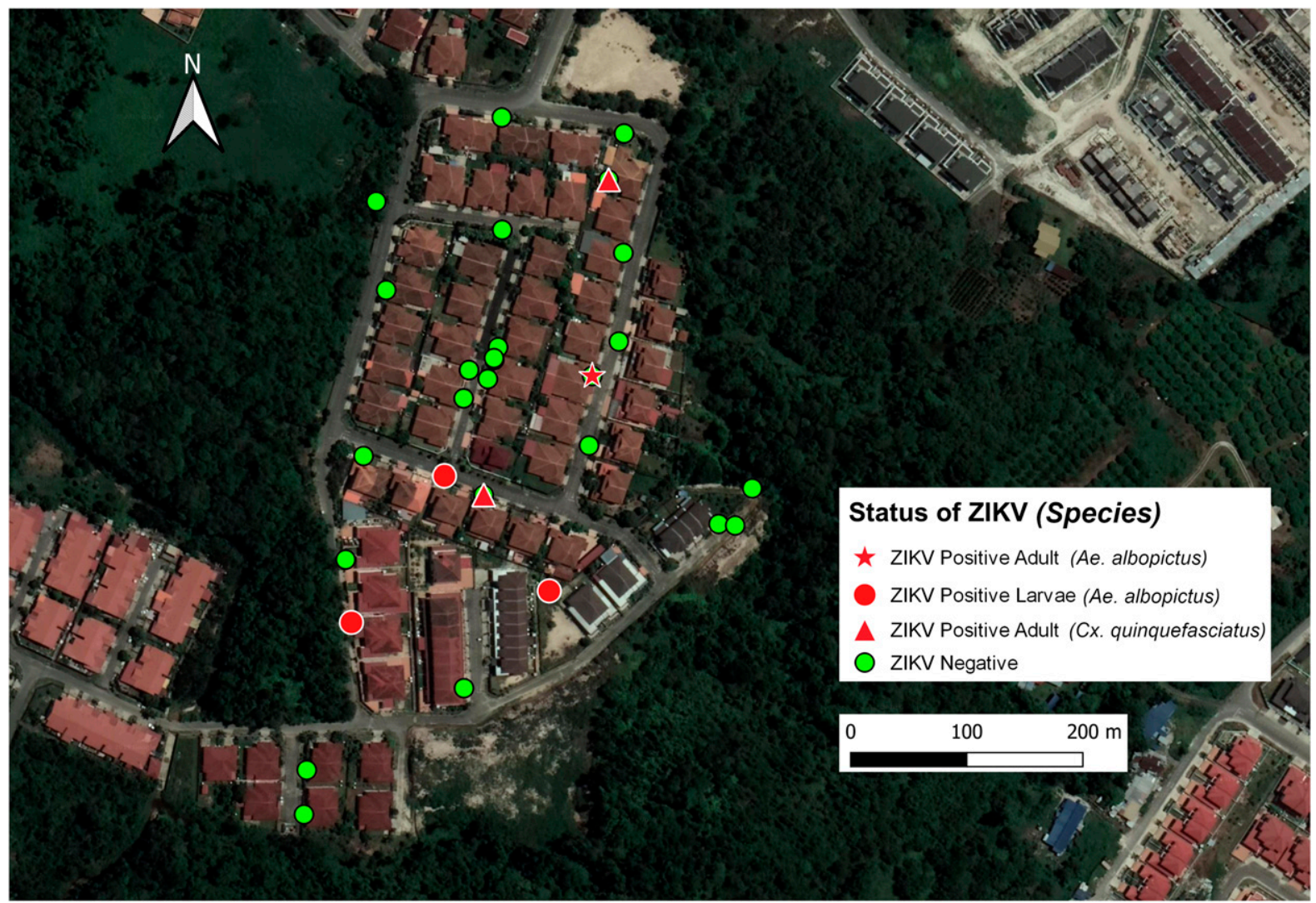

FIGURE 6. Geographical distribution of mosquito species with regard to Zika virus detection in Shang Ri La. 
ZIKV has been detected from a pool of wild-caught mosquitoes in Gabon and Brazil. Moreover, laboratory research has showed that Ae. albopictus is a potential vector of ZIKV in Singapore. ${ }^{20}$ Molecular analysis showed the association of ZIKV with high numbers of Ae. albopictus mosquitoes obtained in this study, whereas none of the Ae. aegypti caught was positive with the virus tested.

It was noteworthy that only Ae. albopictus species detected with ZIKV were caught and obtained from four types of collection methods including larval collection and ovitrapping. Detection of ZIKV from larval specimens also implies that there is a possibility that transovarian transmission occurred in Ae. albopictus. This mode of transmission has been demonstrated by another study ${ }^{21}$ where Ae. aegypti and Ae. albopictus were orally infected with ZIKV and subsequently reared until the fifth generation. Reverse transcription PCR analysis on adult mosquitoes showed that ZIKV was present in both species although the filial infection rate observed in Ae. aegypti was higher than that in Ae. albopictus. Furthermore, a laboratory study by Li et al. ${ }^{22}$ provided evidence of vertical transmission of ZIKV in two strains of $A$ e. aegypti from China. They showed that there was a high infection rate $(100 \%)$ of ZIKV detected from the eggs of both $A e$ aegypti strains after two gonotrophic cycles. Nevertheless, they also indicated that any infected larvae found from the field does not imply that the larvae would develop into infected adults due to possible transstadial loss of infection during the process of maturity to adult stage.

A study by Thangamnani et al. ${ }^{23}$ also proved that ZIKV could be vertically transmitted in Ae. aegypti. Although previous studies have found other flaviviruses such as dengue, yellow fever, and West Nile viruses can use vertical transmission, ${ }^{14,18}$ laboratory experiments have yet to be conducted to determine the susceptibility status of Malaysian Ae. albopictus toward ZIKV and their vertical transmission status.

In this study, ZIKV was detected in adults of Cx. quinquefasciatus and $C x$. gelidus, indicating that these mosquitoes could possibly be the potential vectors. This finding concurs with the study conducted by Phumee et al., ${ }^{24}$ which found $C x$. quinquefasciatus and other species obtained from the field were positive with ZIKV through RT-PCR analysis. The study also detected ZIKV in different developmental stages, that is, larva, male, and female adults of $C x$. quinquefasciatus. However, our study reported that ZIKV was only detected in the adults of $C x$. quinquefasciatus. It is to be noted that the detection of ZIKV from non-Aedes mosquitoes could be due to their incidental infection from feeding on ZIKV patients and the presence of remnants of ZIKV RNA. Furthermore, the RT-PCR technique can only detect genetic materials of the ZIKV and cannot determine the viability of the virus, which will require culturing the virus in cell culture. To incriminate the vector status of $C x$. quinquefasciatus, laboratory infection and epidemiological association studies need to be conducted to better understand the ZIKV vector status of $C x$. quinquefasciatus.

Received May 1, 2019. Accepted for publication December 2, 2019.

Published online March 30, 2020.

Acknowledgments: We would like to thank the Director General of Health Malaysia and the director, Institute for Medical Research (IMR) for their permission to publish this article.

Financial support: This work was supported by the Ministry of Health Malaysia Research Grant (NMRR-16-706-30254).
Authors' addresses: Roziah Ali, Ruziyatul Aznieda Azmi, Nazni Wasi Ahmad, Azahari Abd Hadi, Khairul Asuad Muhamed, Rosilawati Rasli, Cheong Yoon Ling, and Han Lim Lee, Medical Entomology Unit, Institute for Medical Research, WHO Collaborating Centre for Vectors, Kuala Lumpur, Malaysia, E-mails: roziahali@imr.gov.my, ruziyatul.aznieda@ gmail.com, nazni@imr.gov.my, azahari@imr.gov.my, asuad@imr.gov.my, rosilawati@imr.gov.my, cheongyl@imr.gov.my, and leeh|@imr.gov.my. Henry Anak Chua, Miri District Health Office, Jalan Temenggong Oyong Lawai Jau, Miri, Malaysia, E-mail: henry_chua19@yahoo.com. Kiew Lian Wan, School of Biosciences and Biotechnology, Faculty of Science and Technology, Universiti Kebangsaan Malaysia, Selangor, Malaysia, E-mail: klwan@ukm.edu.my.

\section{REFERENCES}

1. Dick GWA, Kitchen SF, Haddow AJ, 1952. Zika virus (I). Isolations and serological specificity. Trans R Soc Trop Med Hyg 46: 5.

2. Lanciotti RS, Kosoy OL, Laven JJ, Velez, Jason O, Lambert AJ, Johnson AJ, Stanfield SM, Duffy MR, 2008. Genetic and serologic properties of Zika virus associated with an epidemic, Yap State, Micronesia, 2007. Emerg Infect Dis 14: 1232-1239.

3. Krow-Lucal ER et al., 2018. Association and birth prevalence of microcephaly attributable to Zika virus infection among infants in Paraíba, Brazil, in 2015-16: a case-control study. Lancet Child Adolesc Health 2: 205-213.

4. Marchette NJ, Garcia R, Rudnick A, 1969. Isolation of Zika virus from Aedes aegypti mosquitoes in Malaysia *. Am J Trop Med Hyg 18: 411-415.

5. Rudnick A, Lim TW, 1986. Dengue fever studies in Malaysia. IMR Bull 23.

6. Tappe D, Nachtigall S, Kapaun A, Schnitzler P, Günther S, SchmidtChanasit J, 2014. Acute Zika virus infection after travel to Malaysian Borneo. J Neurol Neurosurg Psychiatry 17: 183-188.

7. Prada P, 2016. Research Indicates Another Common Mosquito May be Able to Carry Zika. Reuters. Available at: https://www. reuters.com/article/us-health-zika-brazil/research-indicatesanother-common-mosquito-may-be-able-to-carry-zikaidUSKCNOW52AW. Accessed November 19, 2018.

8. Guo XX et al., 2016. Culex pipiens quinquefasciatus: a potential vector to transmit Zika virus. Emerg Microbes Infect 5: 9.

9. Hart CE et al., 2017. Zika virus vector competency of mosquitoes, Gulf Coast, United States. Emerg Infect Dis 23: 559-560.

10. Fernandes RS et al., 2016. Culex quinquefasciatus from rio de Janeiro is not competent to transmit the local Zika virus. PLoS Negl Trop Dis 10: 1-13.

11. Huang YJS, Ayers VB, Lyons AC, Unlu I, Alto BW, Cohnstaedt LW, Higgs S, Vanlandingham DL, 2016. Culex species mosquitoes and Zika virus. Vector-Borne Zoonotic Di 16: 673-676.

12. Lau KW, Chen CD, Lee HL, Low VL, Moh HHS-A, 2017. Ovitrap surveillance in Sarawak, Malaysia: a comprehensive. Trop Biomed 34: 795-803.

13. Wan-Norafikah O et al., 2017. Mosquito larval surveillance in a rice field in Tanjung Karang, Selangor, Malaysia. Adv Sci Lett 23: 1480-1483.

14. Rozilawati $\mathrm{H}$ et al., 2015. Surveillance of Aedes albopictus Skuse breeding preference in selected dengue outbreak localities, Peninsular Malaysia. Trop Biomed 32: 49-64.

15. Chouin-Carneiro T, Santos FBdos, 2017. Transmission of major arboviruses in Brazil: the role of Aedes aegypti and Aedes albopictus vectors. Shields VDC, ed. Biological Control of Pest and Vector Insects. Rijeka, Croatia: InTech.

16. Chang K et al., 2018. Monitoring and control of Aedes albopictus, A vector of Zika virus, near residences of imported Zika virus patients during 2016 in South Korea. Am J Trop Med Hyg 98: 166-172.

17. Boyer S, Calvez E, Chouin-carneiro T, Diallo D, 2018. An overview of mosquito vectors of Zika virus. Microbes Infect 20: 646-660.

18. Mombo IM, Jiolle D, Fontenille D, Paupy C, Leroy EM, 2014. Zika virus in Gabon (central Africa) - 2007: a new Threat from Aedes albopictus? PLoS Negl Trop Dis 8: 1-6.

19. Fernandes $C$ et al., 2018. Evidence of vertical transmission of Zika virus in field-collected eggs of Aedes aegypti in the Brazilian Amazon. PLoS Negl Trop Dis 12: e0006594.

20. Li MZI, Wong PSJ, Ng LC, Tan CH, 2012. Oral susceptibility of Singapore Aedes (Stegomyia) aegypti (Linnaeus) to Zika virus. PLoS Negl Trop Dis 6: 8. 
21. Ciota AT, Bialosuknia SM, Ehrbar DJ, Kramer LD, 2017. Vertical transmission of Zika virus by Aedes aegypti and Aedes albopictus mosquitoes. Emerg Infect Dis 23: 880-882.

22. Li CX et al., 2017. Vector competence and transovarial transmission of two Aedes aegypti strains to Zika virus. Emerg Microbes Infect 6: 4 .
23. Thangamani S, Huang J, Hart CE, Guzman H, Tesh RB, 2016. Vertical transmission of Zika virus in Aedes aegypti mosquitoes. Am J Trop Med Hyg 95: 1169-1173.

24. Phumee A, Buathong R, Boonserm R, Intayot P, 2019. Molecular epidemiology and genetic diversity of Zika virus from field-caught mosquitoes in various regions of Thailand. Pathogens 8: 30 . 